\section{Study design of Real World Evidence for Treatment of Hyperkalemia in the Emergency Department (REVEAL-ED): a multicenter, prospective, observational study}

\author{
Zubaid Rafique', Mikhail Kosiborod ${ }^{2}$, Carol L Clark ${ }^{3}$, Adam J. Singer ${ }^{4}$, \\ Stewart Turner ${ }^{5}$, Joseph Miller ${ }^{6}$, Douglas Char ${ }^{7}$, W. Frank Peacock ${ }^{1}$ \\ on behalf of the REVEAL-ED study investigators
}

'Ben Taub General Hospital, Baylor College of Medicine, Houston, TX, USA

${ }^{2}$ Saint Luke's Mid America Heart Institute and University of Missouri-Kansas City, Kansas City, MO, USA

${ }^{3}$ Beaumont Hospital-Royal Oak, Royal Oak, MI, USA

${ }^{4}$ Stony Brook School of Medicine, Stony Brook, NY, USA

${ }^{5}$ ZS Pharma, Inc., San Mateo, CA, USA

${ }^{6}$ Henry Ford Hospital, Detroit, MI, USA

${ }^{7}$ Washington University, Saint Louis, MO, USA

Objective Hyperkalemia affects up to 10\% of hospitalized patients and, if left untreated, can lead to serious cardiac arrhythmias or death. Although hyperkalemia is frequently encountered in the emergency department (ED), and is potentially life-threatening, standard of care for the treatment is poorly defined, with little supporting evidence. The main objectives of this observational study are to define the overall burden of hyperkalemia in the ED setting, describe its causes, the variability in treatment patterns and characterize the effectiveness and safety of ED standard of care therapies used in the United States.

Methods This is an observational study evaluating the management of hyperkalemia in the ED. Two hundred and three patients who presented to the ED with a potassium value $\geq 5.5 \mathrm{mmol} / \mathrm{L}$ were enrolled in the study at 14 sites across the United States. Patients were treated per standard of care practices at the discretion of the patient's physician. In patients who received a treatment for hyperkalemia, blood samples were drawn at pre-specified time points and serum potassium values were recorded. The change in potassium over 4 hours and the adverse events after standard of care treatment were analyzed.

Results and Conclusion This article describes the background, rationale, study design, and methodology of the REVEAL-ED (Real World Evidence for Treatment of Hyperkalemia in the Emergency Department) trial, a multicenter, prospective, observational study evaluating contemporary management of patients admitted to the ED with hyperkalemia.

Keywords Hyperkalemia; Potassium; Electrolyte disturbance
eISSN: 2383-4625

Received: 1 February 2017

Revised: 26 March 2017

Accepted: 28 March 2017

Correspondence to: Zubaid Rafique Ben Taub General Hospital, Baylor College of Medicine, 1504 Ben Taub Loop, Houston, TX 77030, USA E-mail: zubaidrafique@gmail.com

This study was presented at Society for Academic Emergency Medicine 2016 meeting, May 10-13, 2016 in New Orleans, LA; American College of Emergency Physicians 2016 meeting, October 15-19, 2016 in Las Vegas, NV, USA.

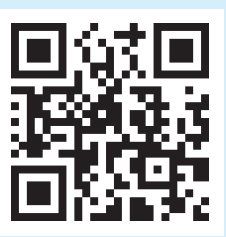

How to cite this article:

Rafique Z, Kosiborod M, Clark CL, Singer AJ, Turner S, Miller J, Char D, Peacock WF. Study design of Real World Evidence for Treatment of Hyperkalemia in the Emergency Department (REVEAL-ED): a multicenter, prospective, observational study. Clin Exp Emerg Med 2017;4(3):154-159.
This is an Open Access article distributed under the terms of the Creative Commons Attribution Non-Commercial License (http:// creativecommons.org/licenses/by-nc/4.0/). 


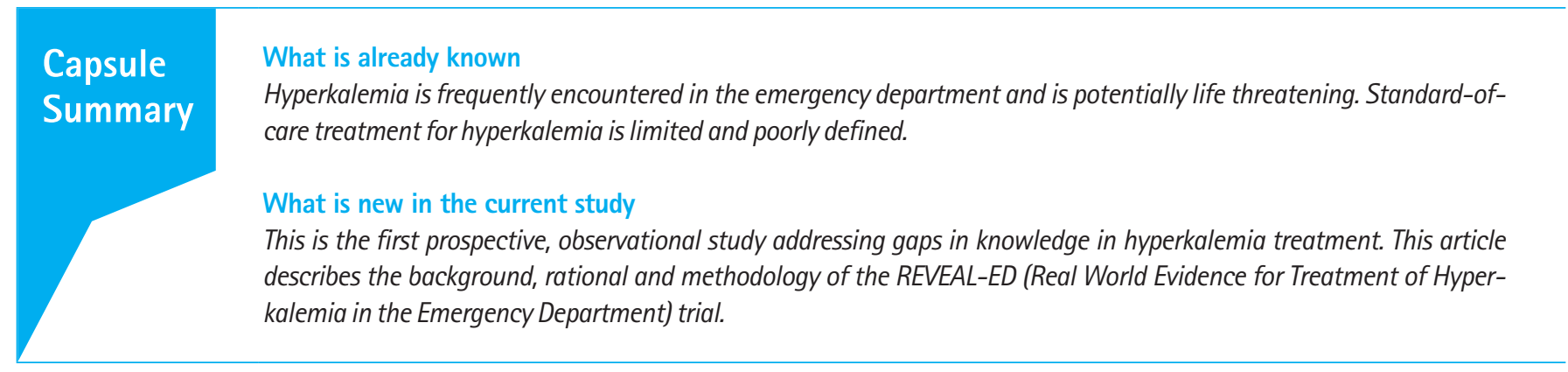

\section{INTRODUCTION}

Hyperkalemia occurs in up to $10 \%$ of hospitalized patients. ${ }^{1-4} \mathrm{~Pa}$ tients with chronic kidney disease and cardiovascular disease, as well as those who are treated with renin-angiotensin-aldosterone system inhibitors, are at greater risk of hyperkalemia., ${ }^{5,6}$ The prevalence of hyperkalemia and related hospitalizations are on the rise in parallel with increased use of renin-angiotensin-aldosterone system inhibitors and an aging hospitalized patient population. Patients are often sent to the emergency department (ED) due to high potassium levels, and over 800,000 hyperkalemia-related ED visits are estimated to occur annually in the US. ${ }^{8}$

If left untreated, hyperkalemia can lead to life-threatening cardiac arrhythmias, ${ }^{9-11}$ and is associated with substantially increased risk of death. ${ }^{12-14}$ One study reported in-hospital mortality of patients with hyperkalemia to be as high as $18.1 \%$, while those with normal potassium to be $3.9 \% .{ }^{13}$ Several other studies have also shown an increase in the risk of death for serum potassium levels above $5 \mathrm{mmol} / \mathrm{L}$ and a rapid increase in the risk of death as serum potassium levels exceed $5.5 \mathrm{mmol} / \mathrm{L}^{6,14,15} \mathrm{In}$ particular, a study by Grodzinsky el al. ${ }^{15}$ reported that in-hospital mortality was greater than $15 \%$ once the maximum potassium level was above $5.5 \mathrm{mmol} / \mathrm{L}$, and another study by Einhorn et al. ${ }^{6}$ reported a 1-day mortality rate up to 17 times higher for hospitalized patients with potassium levels $>6.0$, as compared to $<5.5 \mathrm{mmol} / \mathrm{L}$.

Although no randomized trials on hyperkalemia treatments and their impact on in-hospital mortality have been conducted, available observational data suggest that reducing potassium levels in patients with hyperkalemia may lower mortality risk. For example, an observational cohort study at two medical centers in Korea found that treatment of patients with hyperkalemia with common therapies excluding dialysis, improved serum potassium levels and had a positive association with better survival. ${ }^{16} \mathrm{~A}$ separate observational study of hospitalized patients who received critical care at two tertiary care hospitals in Boston, MA, showed that a reduction of $1 \mathrm{mmol} / \mathrm{L}$ or greater in serum potassium with- in 48 hours after hospitalization was associated with decreased mortality risk. ${ }^{17}$ In addition, a retrospective study of 38,689 consecutive patients with acute myocardial infarction found that inhospital mortality was twice as high among hyperkalemic patients whose potassium levels remained high compared to patients whose potassium levels returned to a normal range. ${ }^{15}$

Although hyperkalemia is frequently encountered in the ED, and is potentially life-threatening, a standard of care (SOC) for hyperkalemia treatment is poorly defined. Acute pharmacologic treatment options for hyperkalemia are limited and their use is complicated by suboptimal tolerability and safety and uncertain efficacy. Hemodialysis is effective for the treatment of hyperkalemia, but is invasive and costly, and significant logistical issues must be overcome in order to initiate this in the ED. In addition, the emergency management of hyperkalemia is not consistent across clinicians and institutions in the US. ${ }^{18} \mathrm{~A}$ Cochrane review of emergency management of hyperkalemia suggested that sufficient randomized, controlled trials supporting the use of commonly prescribed therapies are lacking and many of the trials are methodologically flawed. ${ }^{18}$ Accordingly, we conducted a multicenter, prospective, observational study to define the overall burden of hyperkalemia in the ED setting, describe its causes, and variability in treatment patterns and characterize the effectiveness and safety of ED SOC therapies used in the US. We hypothesized that SOC treatment of hyperkalemia is variable and multiple medications are used in the acute setting. We further hypothesized that all SOC medications are effective in the first four hours of treatment. To our knowledge, this is the first prospective, rigorously designed observational study directly addressing gaps in knowledge in the US.

\section{METHODS}

\section{Study design}

The Real World Evidence for Treatment of Hyperkalemia in the Emergency Department (REVEAL-ED) is a multicenter, prospec- 


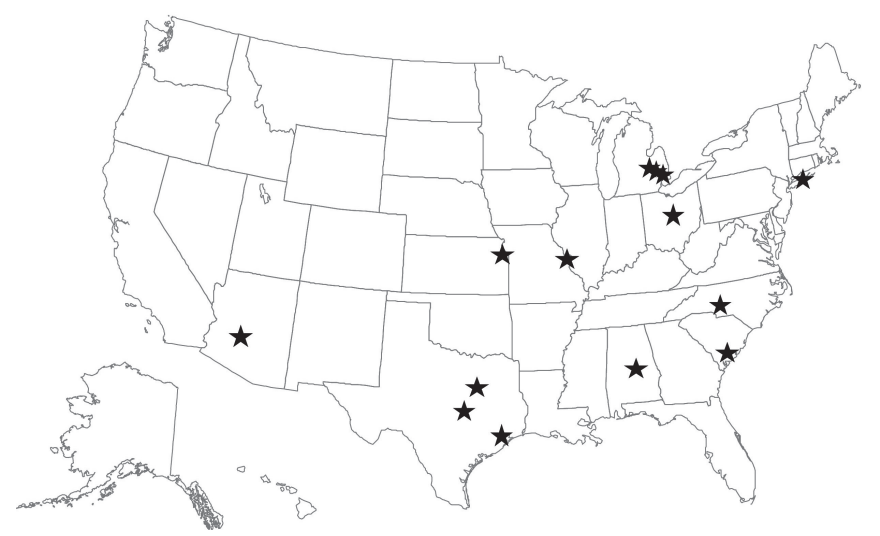

Fig. 1. REVEAL-ED (Real World Evidence for Treatment of Hyperkalemia in the Emergency Department) study sites. Marked areas on the map represent study sites in the US.

tive, observational study evaluating the management of patients admitted to the ED with hyperkalemia. The study was approved by the institutional review board or local ethics committee for each site and registered at ClinicalTrials.gov (NCT02607085). Each patient, legally authorized guardian, or a person with legal responsibility for the patient's health care decisions provided written informed consent prior to participation in any study activities.

\section{Study setting and population}

From October 25, 2015 to March 30, 2016, a total of 203 patients who presented to the ED with a potassium value $\geq 5.5 \mathrm{mmol} / \mathrm{L}$ were enrolled in the study at 14 sites across the US (Fig. 1). Eligible patients were $\geq 18$ years of age, provided written informed consent, and had hyperkalemia confirmed in the ED with a documented potassium $\geq 5.5 \mathrm{mmol} / \mathrm{L}$. Enrollment of patients with a baseline potassium $<6.0 \mathrm{mmol} / \mathrm{L}$ was limited to 50 patients, after which the entry criteria required a baseline potassium $\geq 6.0 \mathrm{mmol} / \mathrm{L}$ A point-of-care analyzer was allowed to determine the baseline potassium level if this was the standard practice at that site. Patients were excluded from the study if, in the opinion of the treating physician, they were unable to perform the tasks associated with the protocol, they were participating in another clinical study which could impact the REVEAL-ED study or they had been previously enrolled in this study.

\section{Study protocol}

After patients provided written informed consent, their demographics and medical history data, including previous ED visits and hospital admissions for hyperkalemia, were recorded. Patients with a prior history of heart failure were classified according to the New York Heart Association functional classification system.

The baseline study-related whole blood potassium was deter-

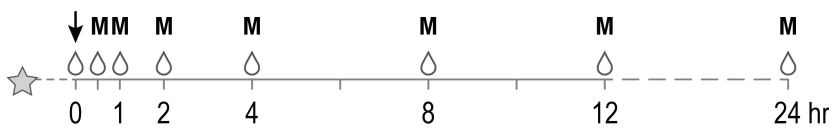

$\widehat{W}$ Screening: (1) Standard of care potassium, (2) subject's chief complaints upon arrival at emergency department, (3) assess possible cause of hyperkalemia, and (4) New York Heart Association assessment if history of heart failure.

$\downarrow$ Initial intervention/treatment

$\checkmark$ Blood draws to measure potassium levels

M Concomitant medications assessment

Fig. 2. Study design.

mined using a point-of-care device (i-STAT; Abbott Point of Care, Princeton, NJ, USA), provided by the sponsor, prior to the first intervention for hyperkalemia. If no baseline study-related potassium value was obtained, then the potassium value qualifying the patient for study entry was considered as the patient's baseline. If the SOC potassium value qualifying the patient for the study was $\geq 5.5 \mathrm{mmol} / \mathrm{L}$, but the baseline i-STAT value was $<5.5 \mathrm{mmol} / \mathrm{L}$ then the SOC value was recorded and the patient was allowed to remain in the study.

In patients who received a treatment for hyperkalemia, studyrelated potassium samples were drawn and analyzed by i-STAT at $0.5,1,2,4,8,12$, and 24 hours after intervention initiation (Fig. 2). If a patient was transferred from the ED to another hospital location, then research staff continued to collect study-related iSTAT samples to the extent feasible. If a patient received more than one intervention for hyperkalemia, then the post-treatment potassium measurement schedule started from the initiation of the first intervention, except if a patient received sodium polystyrene sulfonate, then additional study-related potassium samples were drawn at 1, 2, and 4 hours following initial sodium polystyrene sulfonate administration.

In patients who received no intervention during the initial 4-hour period, study-related potassium samples were drawn and analyzed by i-STAT at 4, 8, 12, and 24 hours after the SOC potassium measurement qualifying the patient for study entry. If a patient was transferred from the ED to another hospital location, then the site continued to collect study-related i-STAT samples to the extent feasible.

Patients were treated per SOC practices at the discretion of the patient's physician. Any medication administered as part of SOC practice was provided according to the drug dispensing policy at each investigational site. No specific treatment algorithms were prescribed by the study protocol, and no medications were supplied by the sponsor during the study. Available data obtained as part of ED SOC management were recorded upon ED admission and up to 24 hours after the initial intervention, or through 24 
hours after the potassium measurement that qualified the patient for entry into the study if no intervention was performed. Available SOC management data that was recorded included physical examinations, vital signs, fluid intake and urine output, electrocardiograms, clinical laboratory data, and results of chest X-rays. Data regarding the patient's chief complaint upon ED admission, possible cause of the patient's hyperkalemia, as well as admitting and discharge diagnoses were recorded. The patient's overall discharge summary and hospital discharge time were also collected when possible.

The timing of each hyperkalemia intervention following ED admission as well as the dose and route of administration were recorded. Concomitant medications were collected for the 14 days before ED admission through ED discharge. If a patient was admitted to another hospital location (i.e., to an in-patient bed), medications administered after ED evaluation was collected for up to seven days or until discharge from the hospital, whichever came first.

Dates and times were recorded for the following (if applicable): ED admission, hospital admission, intensive care unit admission, observation unit admission, step down unit admission, regular floor admission, discharge (from all admissions), dialysis, do not resuscitate order entry, death, and any other recordable outcome deemed significant by the investigator.

Recordable outcomes were limited to pulmonary edema, ventricular tachycardia/fibrillation, pulseless electrical activity arrest, new clinically significant electrocardiogram changes (specifically including but not limited to severe bradycardia, advanced heart block, bundle branch block, tachycardia [> 100 bpm]), palpitations, hypoglycemia, and gastrointestinal-related events (e.g., nausea, vomiting, diarrhea) and any other event deemed significant by the investigator. Recordable outcomes requiring positive-pressure ventilation, central venous access, intubation, chest compressions, intravenous (IV) vasopressors, IV vasodilators, IV anti-arrhythmics, and/or emergency dialysis or resulting in death were also collected. These outcomes were recorded from the time of ED admission through ED discharge. If a patient was admitted to another hospital location, post-ED recordable outcomes were collected for up to 7 days following admission to that unit or until hospital discharge, if earlier. Recordable outcomes resulting in death while the patient remained in the hospital were collected for up to 30 days after ED admission.

\section{Outcome measures}

The primary endpoint of the study was the absolute change in potassium over 4 hours following the initial intervention for hyperkalemia. If a patient did not receive an intervention for hyperkalemia during the ED admission, then the change over 4 hours following the baseline potassium measurement was used.

Secondary endpoints included the rate of change in potassium over 4 hours following the initial intervention for hyperkalemia, change in potassium at other time points (rate, percent and absolute), choice of intervention, timing and details of procedures relative to ED admission, other outcome events (e.g., hospital and intensive care unit admissions, cardiac arrhythmias and conduction abnormalities, hemodynamic instability/cardiac arrest, inhospital deaths), as well as safety and tolerability of SOC interventions were also evaluated.

\section{Data analysis}

No formal sample size calculation was performed for this study. A sample size of 200 patients was selected based on clinical judgement and was considered sufficient to adequately characterize the different interventions in this population. The study populations for analysis include the intent-to-treat and safety populations. The intent-to-treat population included all patients enrolled in the study with any post-baseline study-related potassium values. The safety population included all patients enrolled in the study who had any post-baseline follow-up for safety.

Changes in study-related potassium values following an intervention were used to assess efficacy. All analyses were based on the study-related potassium results obtained using i-STAT. Safety was evaluated using recordable outcomes, clinical laboratory parameters, vital signs, fluid intake and urine output, physical examination, electrocardiograms, and chest X-rays. The principle of treatment emergence was employed for the analysis of recordable outcome data. Treatment emergence was defined to be any event that occurred during the observation period of the study and was not present at baseline, or one that represents an exacerbation of a condition present at baseline.

Recordable outcomes were classified by the medical dictionary for regulatory activities (MedDRA). The type, incidence, timing (onset and duration), relationship to hyperkalemia and to intervention for hyperkalemia, and severity of recordable outcome was reported for treatment-emergent outcomes. Reasons for withdrawal due to recordable outcomes were also reported. The incidence of clinically significant cardiac events (e.g., cardiac arrhythmia, cardiac arrest, cardiovascular and all-cause death) was calculated. All cardiac events were adjudicated by an independent review committee prior to database lock using available SOC data for each case. Descriptive statistics will be presented overall for the study population, as well as stratified by potassium levels at baseline, and by treatment group. When appropriate, hierarchical multivariable analyses will be performed adjusting for differences in patient characteristics and hospital site. 


\section{Role of the sponsor}

The study sponsor participated in developing the study design, conducted data collection, and will participate in data analyses. The study investigators and all authors provided critical input for the study design and methodology, will have free access to the study data, will lead the analysis and interpretation of the data, and have sole discretion in the writing of the report and the decision to submit the manuscript for publication.

\section{DISCUSSION}

Hyperkalemia is frequently encountered in the ED and can lead to cardiac arrhythmias or death. Emergency management of hyperkalemia and close monitoring in the hospital setting is often necessary, but SOC for hyperkalemia has not been well characterized. To our knowledge, this is the first prospective, rigorously designed observational study directly addressing the gaps in knowledge of hyperkalemia management in the United States.

A few noteworthy points about our methodology are as follows: First, our primary end point is to measure the effect of an emergent intervention in the first 4 hours. This time-line was chosen to evaluate how quickly the SOC intervention is effective, as severe hyperkalemia can be lethal and quick interventions are needed in the emergency setting. Moreover, this time frame made the study feasible to conduct in the ED setting. Second, we enrolled only fifty patients with a potassium range $\geq 5.5 \mathrm{mmol} / \mathrm{L}$ and $<6.0$ $\mathrm{mmol} / \mathrm{L}$ because we wanted to see the effectiveness of the interventions at higher potassium levels. Lastly, even though this was an ED based study and we were interested in the effectiveness of interventions in the first 4 hours, we collected data up to 7 days after admission, when applicable, so we could evaluate some of the lasting side effects of the SOC interventions.

This study has several limitations inherent to the nature of an observational study design. For instance, patients were not randomized to the different interventions since they were treated per SOC practices at the discretion of the patient's physician. Also, the medications administered as part of SOC practice were not standardized across investigational sites as medications were provided according to the hospital standards at each investigational site. Hence, we expect to find different dosages of the same medication being used at different sites. The observational design of the study allows for an accurate description of the current SOC therapies used to treat hyperkalemia in the US, but the study is not designed to directly compare the efficacy of these interventions.

In summary, the REVEAL-ED study seeks to define the overall burden of hyperkalemia in the ED setting and will describe the variability in treatment patterns as well as characterize the effectiveness and safety of ED SOC therapies used to treat hyperkalemia in the US.

\section{CONFLICT OF INTEREST}

No potential conflict of interest relevant to this article was reported.

\section{ACKNOWLEDGMENTS}

This study was sponsored and funded by ZS Pharma, Jessica Mendoza, PhD, an employee of ZS Pharma provided editorial assistance for the preparation of this manuscript. David Morris of Webb Writes provided statistical support for this study and received consulting fees from ZS Pharma for this work.

\section{REFERENCES}

1. Stevens MS, Dunlay RW. Hyperkalemia in hospitalized patients. Int Urol Nephrol 2000;32:177-80.

2. Moore ML, Bailey RR. Hyperkalaemia in patients in hospital. N Z Med J 1989;102:557-8.

3. Paice B, Gray JM, McBride D, Donnelly T, Lawson DH. Hyperkalaemia in patients in hospital. Br Med J (Clin Res Ed) 1983; 286:1189-92.

4. Shemer J, Modan M, Ezra D, Cabili S. Incidence of hyperkalemia in hospitalized patients. Isr J Med Sci 1983;19:659-61.

5. Jain N, Kotla S, Little BB, et al. Predictors of hyperkalemia and death in patients with cardiac and renal disease. Am J Cardiol 2012;109:1510-3.

6. Einhorn LM, Zhan $M$, Hsu VD, et al. The frequency of hyperkalemia and its significance in chronic kidney disease. Arch Intern Med 2009;169:1156-62.

7. Juurlink DN, Mamdani MM, Lee DS, et al. Rates of hyperkalemia after publication of the Randomized Aldactone Evaluation Study. N Engl J Med 2004;351:543-51.

8. Aggarwal S, Topaloglu H, Kumar S. Trends in emergency room visits due to hyperkalemia in the United States. Value Health 2015;18:A386.

9. Freeman $K$, Feldman JA, Mitchell $P$, et al. Effects of presentation and electrocardiogram on time to treatment of hyperkalemia. Acad Emerg Med 2008;15:239-49.

10. Fisch C. Relation of electrolyte disturbances to cardiac arrhythmias. Circulation 1973;47:408-19.

11. Surawicz B. Relationship between electrocardiogram and electrolytes. Am Heart J 1967;73:814-34. 
12. Krogager ML, Torp-Pedersen $\mathrm{C}$, Mortensen RN, et al. Shortterm mortality risk of serum potassium levels in hypertension: a retrospective analysis of nationwide registry data. Eur Heart J 2017;38:104-12.

13. Conway R, Creagh D, Byrne DG, O'Riordan D, Silke B. Serum potassium levels as an outcome determinant in acute medical admissions. Clin Med 2015;15:239-43.

14. Goyal A, Spertus JA, Gosch $K_{\text {, }}$ et al. Serum potassium levels and mortality in acute myocardial infarction. JAMA 2012;307: 157-64.

15. Grodzinsky A, Goyal A, Gosch K, et al. Prevalence and progno- sis of hyperkalemia in patients with acute myocardial infarction. Am J Med 2016;129:858-65.

16. An JN, Lee JP, Jeon HJ, et al. Severe hyperkalemia requiring hospitalization: predictors of mortality. Crit Care 2012;16:R225.

17. McMahon GM, Mendu ML, Gibbons FK, Christopher KB. Association between hyperkalemia at critical care initiation and mortality. Intensive Care Med 2012;38:1834-42.

18. Mahoney BA, Smith WA, Lo DS, Tsoi K, Tonelli M, Clase CM. Emergency interventions for hyperkalaemia. Cochrane Database Syst Rev 2005:CD003235. 